\title{
Resonant control of solitons
}

\author{
S.V. Batalov ${ }^{\mathrm{a}, \mathrm{b}}$, A.G. Shagalov ${ }^{\mathrm{a}, \mathrm{b}, *}$ \\ a Institute of Metal Physics, S. Kovalevskaya 18, Ekaterinburg 620990, Russian Federation \\ ${ }^{\mathrm{b}}$ Ural Federal University, Mira 19, Ekaterinburg 620002, Russian Federation
}

\section{A R T I C L E I N F O}

\section{Article history:}

Received 29 November 2012

Received in revised form 21 January 2013

Accepted 23 January 2013

Available online 4 February 2013

Communicated by V.M. Agranovich

\section{Keywords:}

Control

Envelope solitons

Scattering on resonance

Driven nonlinear Schrödinger equation

\section{A B S T R A C T}

It is shown that the effect of "scattering on resonance" can be used to control envelope solitons in the driven nonlinear Schrödinger equation. The control occurs by the frequency modulated driving with multiple crossing of the resonant frequency of the soliton.

Crown Copyright (C) 2013 Published by Elsevier B.V. All rights reserved.

\section{Introduction}

Control of envelope solitons and, in particular, amplification of solitons, is a fundamental problem in nonlinear optics [1,2], spin waves in magnetics [3,4] and another applications in condensed matter physics [5].

An effective method to excite and control envelope solitons was proposed in Refs. [6-8]. The method was based on the autoresonant phenomenon when the soliton was captured by the resonant driving with the frequency close to the internal frequency of the soliton. However, the method appears very difficult for applications because the capture of the soliton of a finite amplitude occurs only in a narrow range of the driving frequencies and with strong restrictions on the phase of the driving [7]. In this Letter we propose that, in contrast to the autoresonance, the promising method of control and amplification of solitons can be based on the "scattering on resonance" [9-11] without capture of the soliton. The proposed method overcomes strong restrictions on the driving which are required to control solitons in the autoresonant approach.

Consider the equation

$i u_{t}+\frac{1}{2} u_{x x}+|u|^{2} u=\varepsilon e^{i \psi(t)}, \quad 0<\varepsilon \ll 1$,

where the small oscillating driving in the right hand side has a slowly varying frequency: $\Omega(t)=\psi_{t}(t), \Omega_{t} \sim \varepsilon$. The perturbation

\footnotetext{
* Corresponding author at: Institute of Metal Physics, S. Kovalevskaya 18, Ekaterinburg 620990, Russian Federation.

E-mail addresses: svbatalov@imp.uran.ru (S.V. Batalov), shagalov@imp.uran.ru (A.G. Shagalov).
}

of this type appears for soliton propagation in twin core optical fibers [12], forced optical ring cavities [13], driven waves in plasmas [14] and magnets [15], condensates [16] and similar cases associated with the small amplitude limit of the sine-Gordon equation in ac-driven Josephson junctions [17] and easy-plane magnets [18].

If $\varepsilon=0$, Eq. (1) has the soliton solution

$u_{s}=\frac{a e^{i \theta}}{\cosh \left[a\left(x-\xi_{0}\right)\right]}, \quad \theta=\omega t+\theta_{0}$,

where $\xi_{0}$ and $\theta_{0}$ are the coordinate and the initial phase of the soliton, $a$ is its amplitude and

$\omega=a^{2} / 2$

is the internal frequency of the soliton. If the varying frequency of the driving crosses the resonance with the soliton frequency $\Omega(t) \approx \omega$, the effect of the perturbation becomes strong and, in spite of a small perturbation, the amplitude of the soliton may be varied significantly. In this Letter we demonstrate that the resonant effect can be applied to control solitons for a special variation of the driving frequency $\Omega(t)$. One notes that another approach to control solitons when the driving frequency is much greater than the resonant frequency of the soliton $\Omega \gg \omega$ was studied in Ref. [19].

\section{Equations for soliton parameters}

Variations of the soliton parameters in the perturbed Eq. (1) obey the following equations 
$\delta_{t}=\Delta \Omega-\frac{\varepsilon \pi a}{\Omega} \cos \delta$,

$a_{t}=-\frac{\varepsilon \pi a^{2}}{2 \Omega} \sin \delta$,

which have been found in Ref. [7]. Here, $\delta=\theta-\psi$ is the phase shift between the soliton and the driving,

$\Delta \Omega(t)=a^{2} / 2-\Omega(t)$

is the difference in frequencies of the soliton and the driving. Eqs. (4), (5) were obtained by adiabatic Lagrangian approach similar to Ref. [20] which used the reduced variational problem $\delta \int L d t=0$. Here, $L=\int_{-\infty}^{+\infty} \mathcal{L} d x$ with the Lagrangian [7] $\mathcal{L}=\frac{1}{2}\left[i\left(u u_{t}^{*}-u^{*} u_{t}\right)-\left|u_{x}\right|^{2}-|u|^{4}\right]-|u|^{2}\left(u \chi^{*}+u^{*} \chi\right), \chi=$ $-(\varepsilon / \Omega) e^{i \psi(t)}+O\left(\varepsilon^{2}\right)$, where, in the adiabatic approximation, we used the soliton parameter function $u=u_{s}(a, \theta)$ of the form (2).

We shall suppose that the driving frequency depends on time linearly

$\Omega(t)=\Omega(0)-\alpha t, \quad \alpha \sim \varepsilon$.

Let us consider a frequency range near the resonance when

$\Delta \Omega \sim \sqrt{\varepsilon}$.

Variations of the soliton parameters in this range will be slow and it is convenient to introduce a "slow" time $\tau=\sqrt{\varepsilon}$. Solutions of Eqs. (4), (5) can be written as series

$\delta(\tau)=\delta_{0}(\tau)+\sqrt{\varepsilon} \delta_{1}(\tau)+\varepsilon \delta_{2}(\tau)+\cdots$,

$a(\tau)=a_{0}+\sqrt{\varepsilon} a_{1}(\tau)+\varepsilon a_{2}(\tau)+\cdots$,

$a(0)=a_{0}$

and the driving frequency (7) takes the form

$\Omega=\Omega_{0}+\sqrt{\varepsilon} \Omega_{1}-\sqrt{\varepsilon} \beta \tau, \quad \beta=\alpha / \varepsilon \sim 1$.

Here, $\Omega_{0}=a_{0}^{2} / 2$ is the resonant frequency and the frequency mismatch at the initial time $\tau=0$ takes the form

$\Delta \Omega(0)=\sqrt{\varepsilon} \Omega_{1}$.

Substituting these series into Eqs. (4), (5) one finds in the lowest order

$\delta_{0, \tau}=a_{0} a_{1}-\Omega_{1}+\beta \tau$,

$a_{1, \tau}=-\pi \sin \delta_{0}$

which give the equation for the phase difference

$\delta_{0, \tau \tau}=-\pi a_{0} \sin \delta_{0}+\beta$.

The equation for the phase difference of the form (15) was applied in studying of the autoresonant effect in Ref. [9]. It is the equation for nonlinear pendulum which defines motion of a "quasiparticle" in the potential

$V\left(\delta_{0}\right)=-\pi a_{0} \cos \delta_{0}-\beta \delta_{0}$.

The phase portrait of Eq. (15) is shown in Fig. 1. If the parameter

$\lambda=\frac{\beta}{\pi a_{0}}$

is small enough, $|\lambda| \leqslant 1$, the potential has minimum in $\delta_{0}=\delta_{c}$ and trajectories are divided into closed trajectories inside the separatrix loop and unclosed ones outside the loop. For the closed trajectories, the associated phase difference varies only in the finite range $\delta_{-} \leqslant \delta_{0}(\tau) \leqslant \delta_{+}$, thus, the phase of the soliton is captured by the

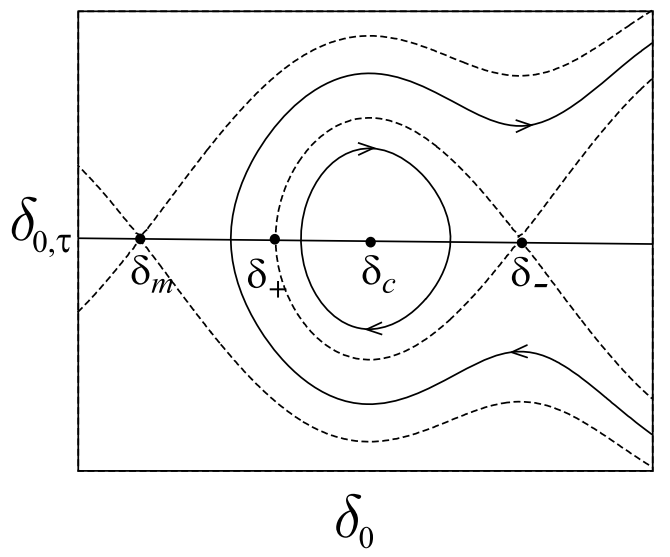

Fig. 1. Phase trajectories of Eq. (15) (solid lines) for $\alpha>0$. Dotted lines - separatrices.

driving. In this case one can control soliton parameters by varying the driving frequency (autoresonant control) [7,9]. Obviously, the capture occurs only when the phase point at the initial time gets into a small domain of the phase space located inside the separatrix loop. It imposes a strong restrictions on the initial parameters of the soliton. It needs to know the amplitude of the soliton with accuracy $\sim \sqrt{\varepsilon}$ to determine correctly the resonant frequency and, in addition, some complex conditions for synchronization of phases of the soliton and the driving should be applied [7]. Both conditions are difficult to satisfy and the capture of a soliton by the driving seems rather exclusive effect in typical experimental conditions. In this Letter we propose the method to control solitons by "scattering on resonance", which is based on dynamics of the system in open trajectories and, thus, the method is opposite to the autoresonance.

\section{Scattering on resonance and soliton control}

The variation in the soliton amplitude for an open trajectory is defined by the integral which follows from Eq. (14),

$\Delta a=-\pi \sqrt{\varepsilon} \int_{-\infty}^{\infty} \sin \delta_{0}(\tau) d \tau$,

where $\delta_{0}(\tau)$ is a solution of Eq. (15). Because of the oscillating integrand, only a segment of the trajectory in the vicinity of the resonant line $\delta_{0, \tau}=0$ gives the main contribution to the magnitude of the integral [21]. This contribution is called "scattering on resonance" $[10,11]$. Let the trajectory crosses the resonant line in the point $\delta_{0}=\delta^{*}\left(\delta_{m}<\delta_{*}<\delta_{+}\right)$at $\tau=0$, then the approximate solution near this critical point is

$\delta_{0}(\tau)=\delta^{*}-C \tau^{2} / 2, \quad C=\pi a_{0} \sin \delta^{*}-\alpha / \varepsilon$,

and integration gives

$(\Delta a)_{0}=-\sqrt{\varepsilon} \pi^{3 / 2}\left(\operatorname{sign}(\alpha) \cos \delta^{*}+\sin \delta^{*}\right) / \sqrt{|C|}$.

Phase trajectories may also approach resonant line in the neighborhood of the saddle points $\delta_{0}=\delta_{-}$and $\delta_{0}=\delta_{m}$. It gives additional contribution to the variation of the amplitude. In the vicinity of the first point in the domain $\delta_{0, \tau}>0$, we suppose $\delta_{0}=\delta_{-}+\xi(\tau)$, $\xi(0)=0$. The solution of Eq. (15) in the linear approximation takes the form

$\xi(\tau)=\frac{\delta_{0, \tau}(0)}{2 \kappa}\left(e^{\kappa \tau}-e^{-\kappa \tau}\right), \quad \kappa^{2}=\pi a_{0} \sqrt{1-\lambda^{2}}$.

The integral also converges on this solution and gives 


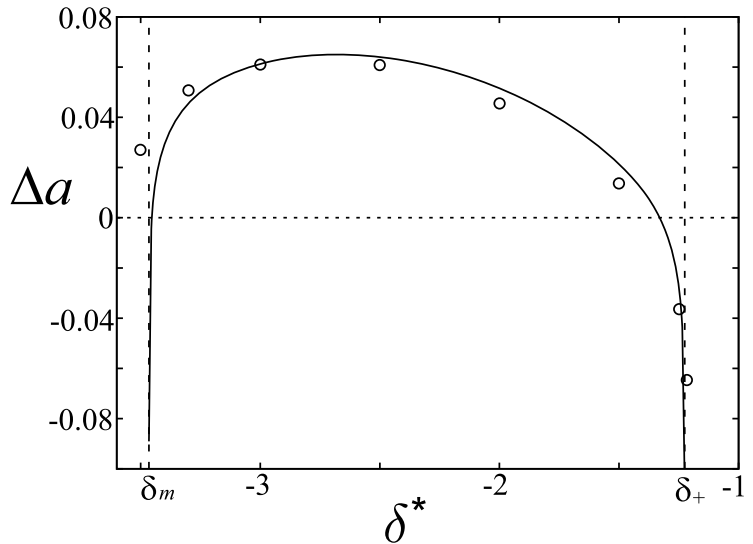

Fig. 2. The dependence of the jump of the soliton amplitude on the parameter $\delta^{*}$ $\lambda=0.318$. Circles - numerical results for the jumps simulated in Eq. (1); $a_{0}=1$, $\alpha=0.001, \varepsilon=0.001$.

$(\Delta a)_{1}=\sqrt{\varepsilon} \frac{2 \pi \lambda}{\kappa} \mathrm{K}_{0}\left(\frac{\delta_{0, \tau}(0)}{\kappa}\right)$,

where $\delta_{0, \tau}(0)$ evaluates via $\delta^{*}$ using conservation of the energy: $E=\delta_{0, \tau}^{2}(0) / 2+V\left(\delta_{-}\right)=V\left(\delta^{*}\right)$. The contribution (20) logarithmically grows when the trajectory passes close to the saddle point $\left(\delta_{0, \tau}(0) \rightarrow 0\right)$ and rapidly decreases as $\exp \left(-\delta_{0, \tau}(0) / \kappa\right)$ when the trajectory passes far off the saddle point.

The similar calculations in the vicinity of the second point $\delta_{0}=$ $\delta_{m}$ give the contribution

$$
\begin{aligned}
(\Delta a)_{2}= & \sqrt{\varepsilon} \frac{\pi^{2}}{\sqrt{A}}\left[\sin \left(\delta^{*}+\frac{C}{A}\right) \mathrm{N}_{0}\left(\frac{|C|}{A}\right)\right. \\
& \left.-\operatorname{sign}(\alpha) \cos \left(\delta^{*}+\frac{C}{A}\right) \mathrm{J}_{0}\left(\frac{|C|}{A}\right)\right],
\end{aligned}
$$

$A=-\pi a_{0} \cos \delta^{*}$

When the trajectory tends to the saddle point $\left(\delta^{*} \rightarrow \delta_{m}\right)$ this value logarithmically grows to $-\infty$ at $\alpha>0$ or to $+\infty$ in the opposite case. The formula (21) is defined only in the range $\delta_{m}<\delta^{*}<$ $-\pi / 2$ for $\alpha>0$, or $\pi / 2<\delta^{*}<\delta_{m}$ for $\alpha<0$. If $\delta^{*} \rightarrow \pm \pi / 2$, one finds $(\Delta a)_{2}=(\Delta a)_{0}$. Bearing in mind this remark, we shall use the following composite formula for the whole jump of the amplitude for $\alpha>0$

$\Delta a= \begin{cases}2(\Delta a)_{1}+(\Delta a)_{2}, & \delta_{m}<\delta^{*}<-\pi / 2, \\ 2(\Delta a)_{1}+(\Delta a)_{0}, & -\pi / 2<\delta^{*}<\delta_{+},\end{cases}$

and for $\alpha<0$,

$\Delta a= \begin{cases}2(\Delta a)_{1}+(\Delta a)_{0}, & \delta_{+}<\delta^{*}<\pi / 2, \\ 2(\Delta a)_{1}+(\Delta a)_{2}, & \pi / 2<\delta^{*}<\delta_{m} .\end{cases}$

In what follows we give a detailed numerical simulation of the soliton control in the main equation (1). We used the standard Fourier transform numerical method (see, e.g. [22]) to solve Eq. (1). We consider the case $\alpha>0$ which is important for applications and corresponds to amplification of solitons. The dependence of the amplitude jump (22) on the parameter $\delta^{*}$ is shown in Fig. 2 by the solid line. Singularities in edges caused by the passage of the trajectory near the saddle points. The circles in the figure are numerical results for variations of the soliton amplitude in Eq. (1) under consistent initial conditions. One notes that the agreement of numerical and theoretical results becomes worse when $\lambda$ decreases. In this case the segment $\left[\delta_{m}, \delta_{+}\right]$contracts and the effect of saddle points becomes predominant. In particular, sometimes the capture of the solitons was observed. It corresponds to the passage of the trajectories across the separatrix toward the interior of the separatrix loop. Similar processes have been studied in

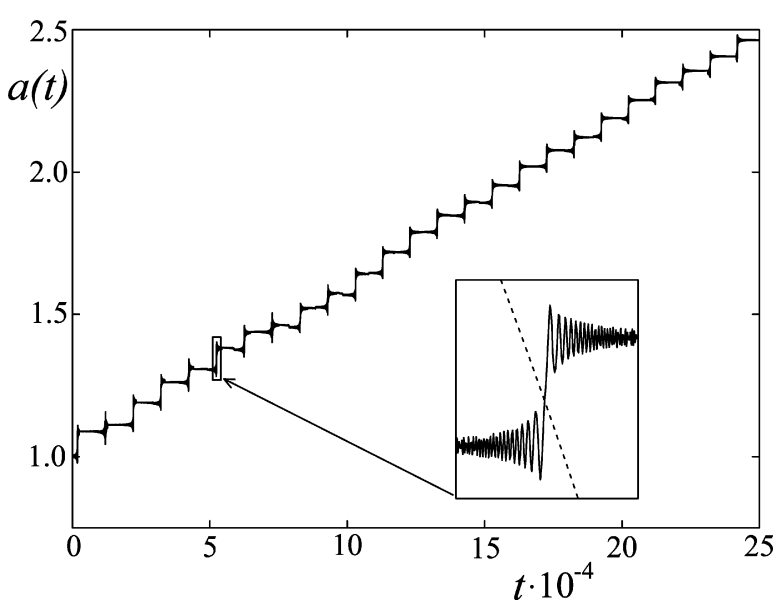

Fig. 3. Numerical simulation of soliton amplification in Eq. (1) with initial amplitude $a(0)=1 ; \alpha=0.00025, \varepsilon=0.0005, \Omega_{\min }=0.4, \Omega_{\max }=3, T=1.04 \cdot 10^{4}$.

Refs. $[10,11]$. Moreover, the separatrix crossing even was proposed to control dynamics of charged particles in plasmas [23]. However, in our approach, the separatrix crossing rather make difficult the control of solitons for a small $\lambda$. One can see in Fig. 2 the segments of the parameter $\delta^{*}$ where the soliton increases $(\Delta a>0)$ or decreases $(\Delta a<0)$ its amplitude. Numerical analysis of Eq. (22) shows that the length of the second segments is very small for $\lambda<0.2$. If $\lambda>0.2$, this length grows monotonically and, for $\lambda \rightarrow 1$, the sizes of the segments in which $\Delta a<0$ and $\Delta a>0$ become equal. This result is very important for the control of solitons. If one supposes that trajectories cross the resonant line equiprobably in the interval $\left(\delta_{m}, \delta_{+}\right)$, then each scattering on resonance will increase the soliton amplitude for $\lambda<0.2$. For the greater $\lambda$, the increasing of the amplitude is possible only with some probability. And when $\lambda$ is close to unit, the increasing and decreasing of the amplitude will have equal probabilities.

The amplitude of the soliton in a single scattering is changed on a small value $\sim \sqrt{\varepsilon}$, therefore, one needs multiple crossing of the resonance for a large effect. We have studied numerically the multiple scattering for an impulse-like ("saw-tooth") modulation of the driving frequency of the form

$$
\begin{aligned}
& \Omega(t)=\Omega_{\max }-\alpha[t-(n-1) T], \quad(n-1) T \leqslant t \leqslant n T, \\
& n=1,2,3, \ldots
\end{aligned}
$$

Here, $n$ is the number of the impulse and $T$ is its period. In a single impulse the frequency is changed in the range from $\Omega_{\max }$ to $\Omega_{\min }=\Omega_{\max }-\alpha T$, which allows one to increase the soliton amplitude by scattering on resonance if the frequency of the soliton gets into this range. The results of the numerical simulation of Eq. (1) are shown in Figs. 3, 4 for different $\lambda$. Fig. 3 shows the step-wise growth of amplitude for the small value of $\lambda=0.16$. It is seen that the every scattering results in increasing of the amplitude on a small value $\sim \sqrt{\varepsilon}$, but the multiple scattering gives the large growth of the amplitude. A single jump of the amplitude is scaled in the window embedded in the figure. Here, the dotted line is the function $\sqrt{2 \Omega(t)}$ and its crossing with the solid line $a(t)$ just corresponds to the resonance: $a^{2}(t) / 2=\Omega(t)$. For the driving with the modulated frequency (24) this process allows to increase the amplitude of the soliton from $a_{\min }=\sqrt{2 \Omega_{\min }}$ to $a_{\max }=\sqrt{2 \Omega_{\max }}$.

For the large $\lambda=0.64$, when both increasing and decreasing of the soliton amplitude are possible, the numerical results are shown in Fig. 4. The embedded graphs show details of the amplitude jumps for the single scatterings of different types. For a given $\lambda$, the jumps with growth of the amplitude has larger probability and, on the average, the amplitude increases. The amplitude 


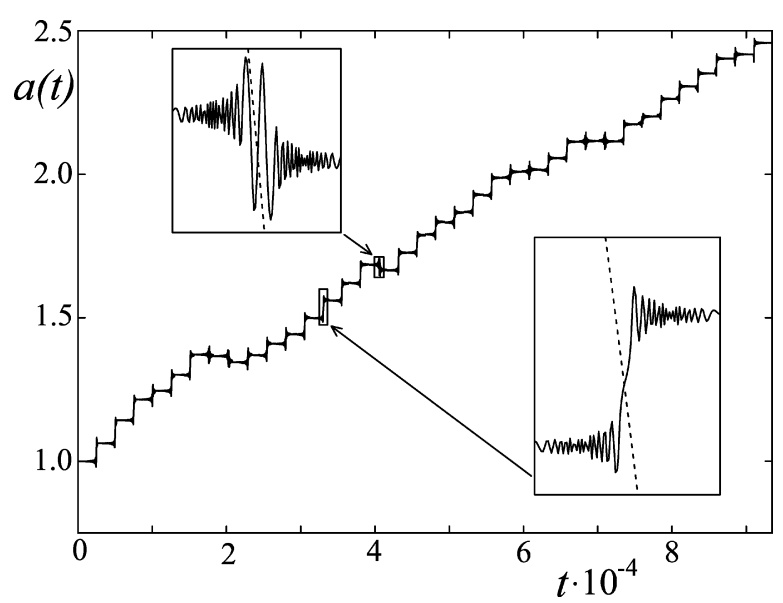

Fig. 4. Numerical simulation of soliton amplification in Eq. (1) with initial amplitude $a(0)=1 ; \alpha=0.001, \varepsilon=0.0005, \Omega_{\min }=0.4, \Omega_{\max }=3, T=2.6 \cdot 10^{3}$.

also can be increased from $a_{\min }$ to $a_{\max }$ as in the previous case of small $\lambda$.

One notes that the process of amplification of the solitons demonstrated good numerical stability which did not depend on the initial phase of the soliton if the value of the parameter $\lambda$ was not too close to the edges of the interval $[0,1]$. For $\lambda \approx 0$, we sometimes observed the captures of the soliton by the driving which result in autoresonant processes with rapid decreasing of the amplitude back to $a \approx a_{\min }$ for the period $T$. On the other hand, for $\lambda \approx 1$, the increasing and decreasing of the amplitude at every scattering are equiprobable and we never observed appreciable changes in the soliton amplitude for a long time. The similar behavior was observed for $\lambda>1$. In this case amplification of the soliton also was not observed, and fluctuations of the amplitude exhibited stochastic behavior at all times of computations.

\section{Conclusions}

In this Letter we have shown that the effect of scattering on resonance can be used for amplification of solitons to large amplitudes. If $\alpha<0$ the process changes trend and the amplitude of solitons, conversely, may be decreased to $a_{\min }$. Therefore, the proposed method allows one both to increase and to decrease the amplitude of the soliton giving technique to control the envelope solitons by the driving with the impulse-like frequency modulation. The crucial condition for the efficient control is $|\lambda|<1$, which restricts the increment of the frequency variation by $|\alpha|<\pi a_{0} \varepsilon$.

\section{Acknowledgement} 1045.

The research was supported by UD RAS, project No. 12-P-2-

\section{References}

[1] H.A. Haus, W.S. Wong, Rev. Mod. Phys. 68 (1996) 423.

[2] Yu. Kivshar, G. Agraval, Optical Solitons: From Fibers to Photonic Crystals, Academic Press, San Diego, CA, 2003.

[3] M.M. Scott, M.P. Kostylev, B.A. Kalinikos, C.E. Patton, Phys. Rev. B 71 (2005) 174440.

[4] A.B. Ustinov, B.A. Kalinikos, V.E. Demidov, S.O. Demokritov, Phys. Rev. B 80 (2009) 052405.

[5] B.A. Malomed, Soliton Management in Periodic Systems, Springer, 2006.

[6] L. Friedland, A.G. Shagalov, Phys. Rev. Lett. 81 (1998) 4357.

[7] S.V. Batalov, E.M. Maslov, A.G. Shagalov, Zh. Eksp. Teor. Fiz. 108 (2009) 1021, Sov. Phys. JETP 108 (2009) 890.

[8] S.V. Batalov, A.G. Shagalov, Phys. Rev. E 84 (2011) 016603.

[9] B.V. Chirikov, Dokl. Akad. Nauk SSSR 125 (1959) 1015, Sov. Phys. Dokl. 4 (1959) 400.

[10] A.I. Neishtadt, Proc. Steklov Inst. Math. 250 (2005) 183.

[11] D.L. Vainchtein, A.I. Neishtadt, I. Mezic, Chaos 16 (2006) 043123.

[12] G. Cohen, Phys. Rev. E 61 (2000) 874.

[13] S. Wabnitz, Opt. Lett. 18 (1993) 601.

[14] K. Nozaki, N. Bekki, Physica D 21 (1986) 381.

[15] S.V. Batalov, A.G. Shagalov, Fiz. Metal. Metalloved. 109 (2010) 3, Phys. Metal. Metallogr. 109 (2010) 1

[16] D.J. Kaup, A.C. Newell, Phys. Rev. B 18 (1978) 5162.

[17] M. Salerno, A.C. Scott, Phys. Rev. B 26 (1982) 2474.

[18] G. Wysin, A.R. Bishop, J. Magn. Magn. Mater. 54 (1986) 1132.

[19] Y.S. Kivshar, K.H. Spatschek, Chaos Solitons Fractals 5 (1995) 2551.

[20] D. Anderson, Phys. Rev. A 27 (1983) 3135.

[21] F.W.J. Olver, Introduction to Asymptotics and Special Functions, Academic Press, New York and London, 1974.

[22] C. Canuto, M.Y. Hussaini, A. Quarteroni, T.A. Zang, Spectral Methods in Fluid Dynamics, Springer Series in Computational Physics, Springer, New York, 1988.

[23] D. Vainchtein, I. Mezic, Phys. Rev. Lett. 93 (2004) 084301. 